Relations industrielles

Industrial Relations

\title{
Réflexions sur les grèves
}

\section{Omer Genest}

Volume 3, numéro 4, décembre 1947

URI : https://id.erudit.org/iderudit/1023584ar

DOI : https://doi.org/10.7202/1023584ar

Aller au sommaire du numéro

Éditeur(s)

Département des relations industrielles de l’Université Laval

ISSN

0034-379X (imprimé)

1703-8138 (numérique)

Découvrir la revue

Citer cet article

Genest, O. (1947). Réflexions sur les grèves. Relations industrielles / Industrial Relations, 3(4), 51-55. https://doi.org/10.7202/1023584ar

Tous droits réservés @ C Département des relations industrielles de l’Université Laval, 1947
Ce document est protégé par la loi sur le droit d'auteur. L’utilisation des services d'Érudit (y compris la reproduction) est assujettie à sa politique d'utilisation que vous pouvez consulter en ligne.

https://apropos.erudit.org/fr/usagers/politique-dutilisation/ 
laisons. La grève du C.C.T. dans la chaussure à Montréal, déclarée en violation des procédures de conciliation et d'arbitrage, est une affaire perdue. Nous ne voulons pas mettre en cause ici les revendications des travailleurs; elles étaient fondées puisque des ajustements ont été accordés. Nous désirons simplement mettre en vedette cette vérité que l'illégalité ne paie pas plus l'union que lemployeur. Des centaines d'unions de la C.T.C.C., du C.M.T. et du C.C.T. ont, par contre, respecté la loi et s'en sont bien trouvées. L'opinion publique supporte encore chez nous, Dieu merci, le régime de la loi et de l'ordre. Qu'on se rappelle les tractations heureuses qui ont suivi les grèves légales à l'Associated Textiles Limited et à la Dominion Textile Company Limited.

Cette rétrospective de l'année syndicale nous amène à conclure que les lois sont sans doute perfectibles mais que la principale réforme reste encore celle des moeurs.

\section{La Direction}

\section{RÉFLEXIONS SUR LES GRÈVES \\ Omer GENEST, ptre}

\section{Une grève, pourvu qu'elle soit LEGALE est-elle MORALEMENT juste? Faut-il condamner en MORALE toute grève ILLEGALE?}

Les grèves, voilà un sujet d'inquiétante actualité. Nous en avons connu, ces dernières années, de fort considérables, de violentes et de paisibles. Récemment, quelques minutes avant l'heure « $G$ », des grèves imminentes furent évitées de justesse, celle des marins, celle des employés de chemin de fer. En France, en Italie, les ouvriers cessent de travailler par centaines de mille, par millions. Les Etats-Unis, après quelque dix-huit mois de paix industrielle vont, selon toute probabilité, subir en 1948 des conflits d'importance par. suite de la décision des unions américaines de réclamer de substantielles augmentations de salaire.

Le fait de la grève produit un effet terrifiant sur les esprits petits-bourgeois qui n'ont jamais pris la peine de comprendre le problème ouvrier. Leur phobie naive du communisme leur fait voir du rouge partout. La peur d'être dérangés dans leur vie calme et douce les porte d'instinct à condamner ceux qui assument les risques d'une lutte pour défendre leurs intérêts menacés. La grève pour eux, c'est la calamité des calamités : l'auteur de l'Apocalypse aurait dû la placer parmi les fléaux qui marqueront la fin du monde.

Qu'il y ait eu des grèves accompagnées de violences, de coups de poing et d'effusions de sang, l'histoire malheureusement l'a enregistré. Mais avant d'en blâmer les ouvriers, il faut rappeler que certains patrons ont eu recours à des tactiques provocatrices, à des mesures de banditisme pour briser l'union de leurs employés qui se défendaient comme ils pouvaient. Plusieurs enquêtes du Sénat américain l'ont prouvé d'une façon irréfutable.

Que des agitateurs aient noyauté le mouvement ouvrier pour le pousser à des cessations de travail injustifiables qui attaquaient sans raison des patrons bien disposés, mais qui n’avaient pas d'autre but que d'être une «initiation réduite, un essai, une préparation du grand bouleversement final » (Georges Sorel). Car dans l'esprit des radicaux, ces grèves en plus d'entretenir le mécontentement populaire, de fomenter des rancours haineuses, entraìnent le prolétariat à s'unir, à se discipliner, afin de pouvoir déclancher un jour la grève générale, premier acte de la révolution qui abattra une structure économique et sociale où «s'accroît la misère, l'oppression, l'esclavage, la dégradation, l'exploitation, » disait Marx, leur maître, dans son «Capital».

Malgré tous ces excès, il faut admettre que, depuis cent ans, les ouvriers ont réussi à améliorer leurs conditions de salaire, de travail et de vie en s'unissant d'abord, puis en maniant l'arme de la grève. Il leur en a beaucoup coûté parfois et la libération de la classe ouvrière dont notre siècle se vante a eu ses martyrs et ses héros. Les grèves, même celles dont l'échec paraissait une capitulation, même les simples menaces de grève * ont cer- 
tainement contribué à faire respecter davantage les ouvriers par les patrons, à prévenir beaucoup d'abus de détail, toutes sortes de mode d'exploitation ou de dégradation », a constaté l'économiste Le Roy Beaulieu et personne ne s'est avisé de trouver qu'il manquait de modération.

La situation a bien changé, et pour le mieux, depuis ces dernières vingt-cinq années surtout. Cependant, les unions ouvrières veulent garder jalousement encore leur droit de déclarer la grève, seule force économique qui reste au nombre organisé pour protéger les intérêts légitimes du travail et se défendre contre des abus toujours possibles du capital. Dans un siècle où le droit sans la force n'est guère reconnu, la classe ouvrière risquerait de revivre de mauvais jours si elle renonçait à la seule force qu'elle possède pour appuyer ses droits.

Il ne faut donc pas voir en toute grève un événement malheureux qui trouble l'ordre social. Il y a grève et grève. Naguère, on parlait de grève juste et des conditions qui rendaient légitime une cessation de travail. Aujourd'hui, l'aspect légal occupe davantage les esprits et tend à servir d'unique critérium pour apprécier un conflit industriel. La question se pose alors : une grève, pourvu qu'elle soit légale, est-elle moralement juste? et d'autre part, faut-il condamner en morale toute grève illégale ?

\section{Aspect moral}

On sait que la grève, c'est la cessation soudaine et générale de tout travail, concertée par un groupe d'employés dans le but d'amener leur patron à mettre fin à certains abus ou à leur consentir de meilleures conditions de salaire et de travail.

Une telle cessation de travail pendant l'existence d'un contrat n'est jamais licite, à moins que l'employeur n'ait violé lui-même l'entente, ou encore que celle-ci ne comporte des clauses sérieusement injustes ou qu'e!le n'ait été signée sous le coup de l'intimidation ou d'une ruse trompeuse. Tout contrat valide doit être respecté même s'il exige des sacrifices, qui ne peuvent être que transitoires et ne durer plus d'un an.

Les grèves de sympathie, qui affectent des patrons non impliqués dans un conflit, ne peuvent être, règle générale, moralement admises. Elles causent un dommage à un employeur qui n'a posé aucun acte répréhensible. Pour la même raison, il faut condamner les grèves qui poursuivent un but politique, c'est-à-dire qui sont une protestation contre telle législation, ou telle ordonnance gouvernementale ou contre la présence de tels hom- mes au pouvoir. En plus du tort qu'elles portent, elles constituent des rébellions larvées contre l'autorité et mènent droit à la guerre civile. La France vient d'en connaître la triste expérience.

Les employés à des fonctions instituées direc. tement pour le bien du pays et dans des entreprises même privées qui pourvoient à des besoins communs de première nécessité ne peuvent légitimement quitter leur travail, à moins que des intérêts d'une importance extrême et très exceptionnels ne soient en jeu et qu'il ne leur reste plus d'autre moyen d'obtenir justice.

Mais des ouvriers, non liés par un contrat, qui ont de légitimes revendications d'ordre professionnel ou économique, qui reçoivent un salaire nettement insuffisant, qui subissent un travail excessif, dans des conditions hygiéniques ou morales déplorables, peuvent certainement recourir à la grève pour mettre un terme à l'exploitation dont ils souffrent. Même s'ils n'avaient pas à se plaindre de pareils abus, ils pourraient le faire pour vaincre la volonté rebelle d'un patron qui refuse de traiter collectivement avec ses employés et s'ingénie à briser leur union; pour obtenir des avantages légitimes, une amélioration raisonnable de leur salaire et de leur travail.

Evidemment, avant de cesser leur travail, les ouvriers doivent avoir épuisé tous les moyens de conciliation et d'entente. Mais si la possibilité d'un accord n'existe pas et que des chances sérieuses de succès appuient une décision périlleuse mais devenue nécessaire, la grève est moralement juste; souvent, elle ne sera pas seulement l'exercice d'un droit, mais un devoir qui oblige tous les intéressés et «comme il y a des déserteurs de l'armée, il peut y avoir aussi des déserteurs de la profession et de la solidarité engendrée par elle» (Mgr Paul Six).

Les grévistes ne peuvent user de moyens violents et injustes pour assurer le triomphe de leur cause; ils doivent respecter la propriété et la personne des employeurs et des autres ouvriers qui ne marcherajent pas avec eux. Contre ceux-ci cependant, ils peuvent employer toute coaction morale légitime; ainsi cette manifestation de la force de l'union aux abords de l'usine qu'on appelle piquetage et dont le but est d'empêcher l'entrée d'ouvriers traîtres à la cause. Il est vrai qu'ici l'on fait intervenir la fameuse liberté du travail et que des générosités candides ou d'hypocrites antiunionistes répètent encore la phrase de Waldeck Rousseau : «Le droit de travailler d'un ouvrier est aussi sacré que le droit de quatre-vingt-dix-neuf à faire la grève.» Formule simpliste qui oublie que la liberté du travail n'est pas une valeur absolue mais 
qu'elle doit se subordonner aux exigences du bien commun et de la profession. Or, il y a des grèves qui sont des devoirs et n'y pas adhérer viole une obligation de solidarité et risque d'occasionner l'échec d'une juste cause entreprise par des confrères de travail.

Voilà donc, en bref, les principes admis par les moralistes pour juger une grève : motifs sérieux et proportionnés, épuisement des moyens d'enten. te, espoir raisonnable de succès, et pendant la grève usage de moyens qui respectent la justice bien comprise. Ces principes sont bien généraux et se tiennent nécessairement dans l'abstraction. La vie réelle prête à beaucoup plus de complications et ces principes ne sont pas toujours faciles à appliquer. Sans compter qu'un facteur nouveau est entré en lice, c'est la législation qui est venue réglementer ou essayer de réglementer l'exercice du droit de grève.

\section{Législation fédérale}

Inutile de rappeler la loi Lemieux (1907) ou la loi des enquêtes en matière de différends industriels (1927) qui avait pour objet d'aider à prévenir ou à régler les grèves et les lock-outs dans les mines et dans les industries se rattachant aux services publics. Le décret du Conseil privé 1003 en contient l'esprit et les grandes lignes. Ce décret, en date du 17 février 1944, s'étendait à toutes les industries et les services jugés essentiels à l'effort de guerre; il ne couvre plus que les cas placés par la Constitution sous la juridiction fédérale.

Les agents négociateurs doivent d'abord obtenir leur accréditation en prouvant qu'ils représentent bien la majorité des employés concernés. Une fois reconnus, ils peuvent demander une rencontre avec l'employeur qui, dans les 10 jours, doit entamer les pourparlers. Si après 30 jours de négociations, l'une ou l'autre partie estime que l'entente sera impossible, elle peut en aviser le Conseil des Relations ouvrières, qui soumettra aussitôt le différend au ministre du Travail. Celui-ci dans les 30 jours doit charger un conciliateur de s'aboucher avec les parties. Après 14 jours, s'il ne parvient pas à les mettre d'accord, il doit faire rapport au Ministre et recommander, s'il le juge bon, l'établissement d'une Commission de Conciliation. Le Ministre en avise aussitôt les parties au différend, qui ont 7 jours pour nommer leur représentant. Si elles ne le font pas, le Ministre s'en charge. Les deux membres désignés ont 5 jours pour choisir un président. S'ils ne s'entendent pas, le Ministre le nomme. La Commission doit faire rapport dans les 14 jours qui suivent la nomination de son pré- sident et transmettre au Ministre ses conclusions et recommandations. Copie du rapport est ensuite envoyée aux parties. Quatorze jours après la soumission du rapport au Ministre, la grève peut être alors déclaree légalement.

Les divers délais prévus depuis la reconnaissance du syndicat jusqu'à la déclaration de la grève forment un total de 107 jours, auxquels il faut ajouter 10 jours pour l'échange de lettres et d'avis nécessaires.

Le bill 338, nouvelle législation fédérale concernant les enquêtes et la conciliation en matière de différends de travail a subi une première lecture à la Chambre des Communes en juin dernier. Il sera discuté et adopté pendant la présente session. Il améliore le décret C.P. 1003 sur certains points importants. Quant à notre sujet, il ne change rien aux prescriptions du décret, sinon qu'il abrège les délais d'une vingtaine de jours. Après l'accréditation du syndicat, les parties ont vingt jours pour s'entendre au lieu de quarante.

Remarquons que les diverses étapes de la procédure ne peuvent traîner indéfiniment. Un délai raisonnable est fixé pour chacune. Le bill 338 a le mérite de les écourter. Cependant, le Ministre, en cas de besoin, peut les prolonger, surtout quand les parties y consentent.

Une fois les recommandations de la Commission connues, les parties sont libres de les accepter ou de les refuser. La seule chose à noter, c'est que 14 jours après le rapport, la grève peut se faire légalement. Les revendications ouvrières sont-elles légitimes, valent-elles le risque des misères fatales inhérentes à une grève, alors seulement celleci est moralement juste. Il est facile de conclure que l'on peut voir une grève parfaitement légale tout en étant totalement immorale.

Il faut regretter que la loi fédérale ne prévoit pas un arbitrage dont les décisions seraient obligatoires quand il s'agit de services publics ou d'industries essentielles au bien commun. C'est une lacune sérieuse dont souffrait le C.P. 1003 et que ne corrige pas le bill 338 .

\section{Législation provinciale}

La législation provinciale a au moins cet avantage marqué que dans les différends affectant les corporations municipales et scolaires, les hôpitaux, les hospices, les centrales téléphoniques ou télégraphiques, les services de transport, d'aqueduc, de gaz et d'électricité, l'arbitrage émet des recommandations qui s'imposent aux deux parties. Sauf les unions ouvrières en mal d'agitation, tous reconnaissent la sagesse d'une pareille loi. Cepen- 
dant, nous sommes forcé d'admettre que l'exemption accordée à certaines commissions scolaires et le droit d'en appeler d'une décision arbitrale auprès de la Commission municipale votés à la dernière session, faussent l'esprit d'une bonne loi et s'avèrent injustifiables.

Les employés des services publics comme tous les autres doivent se soumettre aux procédures établies par la loi des relations ouvrières. L'union reconnue majoritaire par la Commission chargée d'appliquer la loi, donne un avis de 8 jours à l'employeur pour l'informer qu'elle est prête à commencer les négociations. Puis, si après 30 jours, l'entente n'est pas conclue, une des parties peut en avertir la Commission de relations ouvrières qui en informe le Ministre du Travail. Un conciliateur tente de régler le différend. Si ces démarches restent vaines, après 14 jours, il présente son rapport au Ministre qui institue un Conseil d'arbitrage. Les parties prennent tout le temps qu'elles veulent pour désigner leur représentant respectif. Puis ces deux membres choisissent un président. S'ils ne parviennent pas à s'entendre après 10 jours, le Ministre le nomme lui-même.

Le Conseil siège quand et aussi longtemps qu'il veut. Cependant, la dernière instruction terminée, il doit présenter son rapport au Ministre dans les 30 jours. L'union peut, quatorze jours après, déclarer légalement la grève. Car aucune des parties n'est tenue d'accepter les recommandations de l'arbitrage, sauf quand il s'agit d'un différend qui affecte un service public. Que leur cause soit juste ou non, les ouvriers peuvent cesser légalement leur travail.

Telle est la législation provinciale relative aux grèves. Les observateurs constatent que la manière dont elle est appliquée et l'élasticité des délais prévus sont une invitation à faire des grèves illégales.

Un syndicat demande-t-il sa reconnaissance? L'employeur peut s'y opposer, présenter un mémoire à la Commission; le syndicat devra répondre, même comparaître devant la Commission qui étudiera le cas et décidera finalement. De nouvelles représentations peuvent lui être adressées et le débat sera réouvert. Les ouvriers peuvent attendre plusieurs mois avant que leur union soit reconnue et puisse entamer des négociations.

Le patron de mauvaise foi espère gagner du temps et lasser la patience de ses ouvriers qui lâcheront l'union qui ne leur donne rien. Il peut pousser l'audace jusqu'à congédier des officiers de l'union, que tout à coup il découvre incompé- tents, indisciplinés, paresseux. Sa résistance effraiera ses ouvriers, pense-t-il avec raison parfois.

Le conciliateur n'obtient rien. Le Ministre établit alors un conseil d'arbitrage. Le patron peut tarder à désigner son représentant, la loi ne lui fixe aucun délai. Enfin, après un mois ou deux, le conseil est constitué. Il espace ses séances, pour accommoder le délégué du patron qui est fort affairé ailleurs ... Six mois, huit mois, un an, se passent ainsi avant que le Ministre reçoive les décisions de l'arbitrage.

Le patron est en belle posture pour les repousser. Cette longue période d'attente a affaibli le courage des ouvriers, leur confiance dans l'union; un petit jeu habile d'intimidation et de promesse que le patron a pu mener à loisir réussit même à les diviser. Il vaincra facilement la grève qu'une majorité réduite lancera. Au besoin, il engagera des fiers à bras pour briser les lignes de piquetage, s'il ne parvient pas à s'assurer les services de la police pour défendre la fameuse liberté du travail. La grève se prolonge. Un groupe d'employés fondent une association nouvelle, qui réclame le droit exclusif de représenter les employés et qui a les bonnes grâces du patron. La Commission étudie la demande et ira mème jusqu’à reconnaître cette «union de compagnie », qui défendra mollement les intérêts des ouvriers. La patron se réjouit: il a eu tout le temps qu'il a voulu pour écraser l'union sérieuse, effective de ses employés. Il a eu recours à des tactiques déloyales condamnées par la loi. Pourquoi se seraitil gêné ? La menace de poursuite ne s'est jamais réalisée en pareil cas et les pénalités prévues sont inopérantes. D'ailleurs, si l'union avait obtenu le consentement de la Commission ou du Procureur général pour le poursuivre, il aurait sans peine subi l'aventure: de bons avocats auraient plaidé sa cause devant tous les tribunaux du pays, ce qui demande beaucoup de temps et il n'ignore pas que le temps joue en sa faveur.

Alors faut-il être surpris que des unions pour appuyer de justes demandes des ouvriers contre un employeur récalcitrant et sans scrupule, ne prennent pas le risque inutile de suivre les procédures interminables de conciliation et d'arbitrage prévues par la loi des relations ouvrières et se lancent dans une grève illégale tant que l'on voudra, mais qui est le seul moyen sûr de mettre cet employeur à la raison. D’ailleurs, les pénalités qui frappent les grévistes illégaux sont difficilement applicables et n'ont pratiquement jamais été appliquées. Et puis le cas s'est vu de grèves illégales qui ont été rapidement victorieuses. Alors, encore une fois, pourquoi se gêner? Inutile de donner 
des précisions : nous n'avons fait que dégager certaines caractéristiques de conflits industriels survenus ces dernières années pour étayer nos réflexions.

\section{Conclusion}

La loi a pour but de réglementer les activités humaines pour assurer le respect des droits individuels et du bien commun. Il n'est pas normal que d'honnêtes citoyens qui poursuivent un objectif légitime par des moyens justes se voient réduits à désobéir aux lois pour ne pas courir à un échec presque certain. Or c'est le cas des unions ouvrières en face d'un employeur rebelle et de mauvaise foi.

Sans doute, une loi ne peut pas être parfaite du premier coup. La loi des relations ouvrières marquait un progrès dans notre législation. L'esprit qui l'a dictée, malheureusement, n'a pas toujours inspiré son application. Après quelques années d'épreuve, on voit mieux les points qui doivent être améliorés, tant au sujet de ses prescriptions que pour sa mise en oeuvre.

Les reconnaissances syndicales devraient se faire avec plus de rapidité et de méthode en réglant sans retard les contestations qu'elles peuvent soulever et en écartant toute « union de compagnie ».

Les négociations, l'intervention des conciliateurs, les procédures de l'arbitrage doivent se dérouler dans des délais relativement brefs. Ceux prévus par la loi fédérale (bill 338) sont fort acceptables et nous aurions tort de ne pas les inclure dans notre législation provinciale, d'autant plus qu'ils s'appliquent aussi en cas de conflit lors du renouvellement d'une convention collective.

Les employeurs qui se livrent à des tactiques déloyales contre l'union de leurs salariés devraient être poursuivis et punis sans pitié. Il serait alors plus facile d'arrêter avec la même énergie les menées d'agitateurs qui exploitent les malaises ouvriers pour semer des idées fausses, créer des troubles sociaux, fomenter la lutte des classes et le mépris de l'autorité.

Les unions qui veulent collaborer à établir un ordre social dans la justice et la charité, qui recourent à regret mais sans peur quand c'est nécessaire, à l'usage des moyens forts mais honnêtes, et la grève en est un, pour soutenir de légitimes revendications, devraient pouvoir le faire sans risquer que l'obéissance aux lois compromette leur cause.

\title{
UNE SOLUTION COURAGEUSE
}

\author{
W. J. WHITEHEAD \\ directeur-gérant de la Wabasso Cotton Co. Ltd, des Trois-Rivières, Qué.
}

(N. D. L. R. - M. W. J. Whitehead, directeur-gérant de Wabasso Cotton Co. Ltd, Trois-Rivières, explique dans cet artcile les données d'un plan nouveau de participation aux bénéfices dont il est l'auteur et dont jouissent, par convention collective, les 1,400 employés de l'entreprise.)

Il n'est pas besoin de démontrer que le monde industriel, et par voie de conséquences directes, la société toute entière sont bouleversés par l'agitation, les luttes et les incidents ou accidents dans le domaine de la rétribution du travail.

Si l'on se borne à des considérations purement matérielles, il n'y a pas lieu d'être étonné outre mesure quand chacun cherche à vouloir aussi lar- ge que possible sa part des richesses créées par l'industrie, de celles tirées de la terre ou provenant de l'exploitation des ressources naturelles tout en faisant le moins d'effort possible et en comptant sur les gouvernements pour suppléer à ce qui n'a pas été produit par le travail.

Tout cela est bien humain en soi et dans ses manifestations mais amène nécessairement des phénomènes désagréables et nuisibles aux individus et aux collectivités. Cette recherche instinctive de la plus grande part en suivant la ligne du moindre effort, ne peut amener que des conflits d'intérêts, des rivalités de partage s'avérant néfastes quand leur règlement est abandonné aux manoeu. vres, à la force, à la violence ou encore à la ruse; tous moyens aussi purement humains que les sentiments ou les instincts d'où naissent les conflits et les rivalités. 